\title{
Pathology in Practice
}

In collaboration with the American College of Veterinary Pathologists

\section{History}

A 9.5-year-old 37.8-kg sexually intact male Briard was referred to the Purdue University Small Animal Veterinary Teaching Hospital because of pleural effusion, suspected cardiomyopathy, and a 1-month history of coughing, exercise intolerance, hyporexia, and lethargy. Two years prior, the dog had incomplete excision of squamous cell carcinoma (SCC) of the ungual process of distal phalanx on the fourth digit of the right pelvic limb, and more recently, the dog had splenic torsion corrected with splenectomy and a grade II soft tissue sarcoma on the left lateral thorax resected.

\section{Clinical and Clinicopathologic Findings}

On presentation, the dog was cyanotic and dyspneic, with increased respiratory effort. Point of care thoracic ultrasonography revealed a moderate amount of pleural effusion bilaterally. Thoracocentesis was performed for stabilization. Approximately $2.5 \mathrm{~L}$ of red to yellow serosanguinous fluid was removed from the thorax bilaterally and was classified as an exudate (protein concentration, $3.5 \mathrm{~g} / \mathrm{dL}$; total nucleated cell count, 5,060 cells $/ \mu \mathrm{L})$. The dog had a mild regenerative anemia (Hct, 35.3\% [reference range, $37 \%$ to $55 \%$ ]; reticulocyte count, $111.5 \mathrm{X}$ $10^{3}$ cell $/ \mu \mathrm{L}$ [reference limit $<100 \times 10^{3}$ cell $\left./ \mu \mathrm{L}\right]$ ), and the platelets were clumped but estimated to be high based on slide review. Three-view thoracic radiography was performed and revealed moderate pleural effusion with possible pleural thickening or pleural masses, a possible mediastinal mass, and a right caudal lung lobe nodule. Contrast-enhanced CT was performed and revealed marked pleural effusion with parietal, visceral, and mediastinal pleural thickening

Alyssa M. Zoto, DVMl*; Craig A. Thompson, DVM, DACVP2 Lynn P. Little, DVM'; Nolie K. Parnell, DVM, DACVIM'; Garrett S. Oetelaar, DVM'; Caroline V. Fulkerson, DVM, DACVR'; Natalia J. Strandberg, DVM²

'Department of Veterinary Clinical Sciences, College of Veterinary Medicine, Purdue University, West Lafayette, IN

2Department of Comparative Pathobiology, College of Veterinary Medicine, Purdue University, West Lafayette, IN

*Corresponding author: Dr. Zoto (zotoalys@msu.edu)

https://doi.org//0.2460/javma.20.01.0003

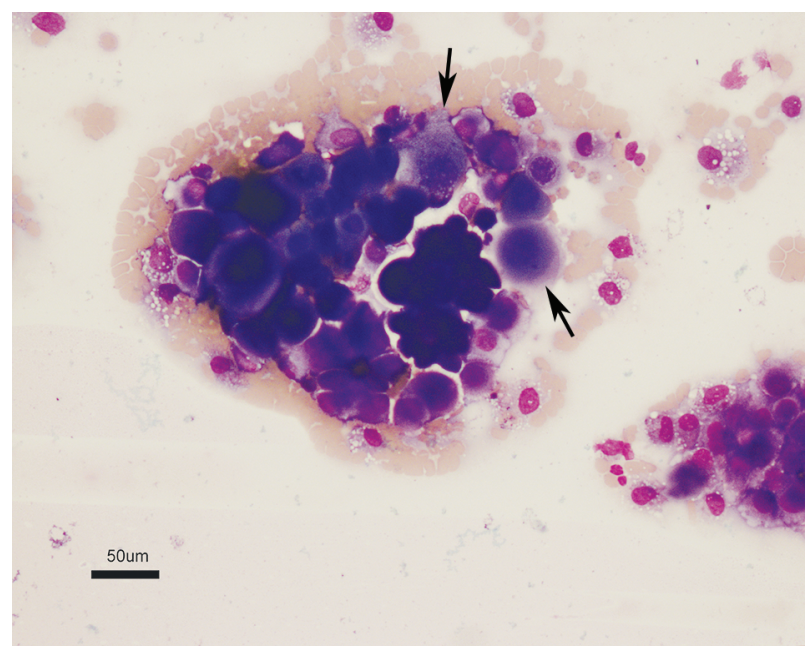

Figure I-Photomicrograph of a thoracocentesis sample of pleural effusion from a 9.5 -year-old $37.8-\mathrm{kg}$ sexually intact male Briard referred because of pleural effusion, suspected cardiomyopathy, and a I-month history of coughing, exercise intolerance, hyporexia, and lethargy. Numerous cells are in small to medium-sized confluent aggregates. The cells appear to be a mixture of vacuolated macrophages, some mesothelial cells, and a few hyalinized epithelial cells consistent with keratinization (arrows). Modified Wright stain; bar $=50 \mu \mathrm{m}$.

and nodules, multifocal mild body wall invasion (fifth and seventh rib osteolysis, xiphoid process periosteal reaction), and pulmonary nodules that raised suspicion for metastatic neoplasia.

Cytologically, the pleural effusion showed a mixed population of nucleated cells arranged in a moderately heavy background of erythrocytes. The nucleated cells were a mixture of neoplastic epithelial cells, several nondegenerate neutrophils, occasional large mononuclear cells (mostly heavily vacuolated macrophages with a few reactive mesothelial cells), and rare small lymphocytes (Figure I). The epithelial cells were small to large in size and round to polygonal in shape. They were seen as singlets and small cohesive clusters and contained a single round to moderately pleomorphic nucleus set in a scant to abundant amount of dark blue to lavender cytoplasm, which occasionally contained several colorless vacuoles, presumed to be keratohyalin granules (Figure 2). The cytoplasm of these cells often took on a ground glass, hyalinized, or keratinized appearance. Additionally, these cells rarely exhibited emperipolesis, with nondegenerate neutrophils 


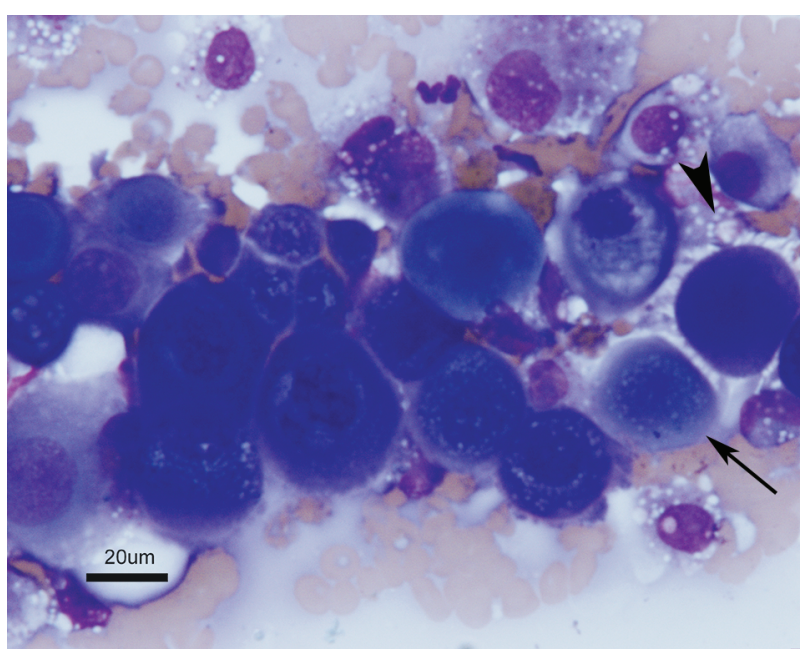

Figure 2-Photomicrograph of several cells in medium-sized confluent aggregate in a sample of pleural effusion from the dog described in Figure I. There are reactive mesothelial cells with blebbing cytoplasm (arrowhead) and nucleated cells that exhibit hyalinized cytoplasm and contain colorless granules consistent with keratohyaline granules (arrow). Modified Wright stain; bar $=20 \mu \mathrm{m}$.

trafficking through their cytoplasm. The nuclei exhibited a coarsely granular chromatin pattern and rarely contained 1 to a few, prominent, variably sized, slightly pleomorphic nucleoli. Marked anisocytosis and anisokaryosis were identified. No mitotic figures were seen. Rare erythrophagia was noted among the macrophages.

Formulate differential diagnoses, then continue reading.

\section{Additional Cytologic and Clinicopathologic Findings}

Findings for this dog were concerning for SCC, and the presence of squamous epithelial cells in a pleural effusion indicated a potential metastatic or malignant process. A fine-needle aspirate sample of the affected lung tissue was submitted for evaluation, and results were cytologically similar to the neoplastic cells seen in the pleural effusion.

Pancytokeratin (AE1/AE3 clone, Agilent) and vimentin (Vim 3b4 clone, Agilent) immunocytochemical staining was performed on the pleural effusion to better classify the neoplasm. The neoplastic population in question showed positive cytoplasmic staining for pancytokeratin but did not stain with vimentin (Figure 3). Cytokeratin and vimentin are special immunohistochemical stains that are especially useful in both human and veterinary medicine for differentiating epithelial and mesenchymal type proliferations. ${ }^{1}$ The cytoplasm of both healthy and neoplastic cells label with these stains, and therefore staining does not differentiate whether the cells in question are malignant. Cytokeratins are intermediate filaments found in epithelial cell types and are

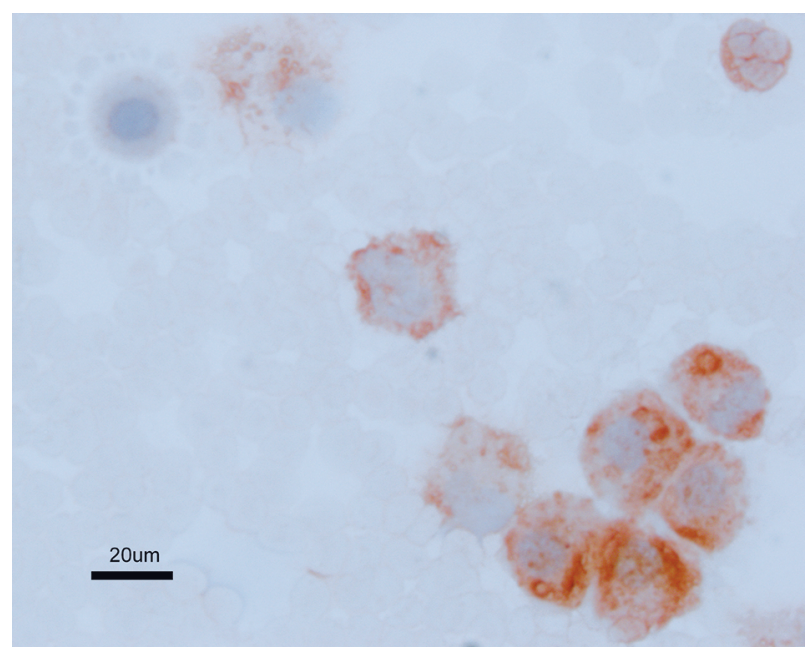

Figure 3-Pancytokeratin immunocytochemical staining of a sample of the pleural effusion from the same dog reveals several nucleated cells that exhibit moderate to marked cytoplasmic positivity. Pancytokeratin immunocytochemical stain (pancytokeratin mouse anti-human cytokeratin antibody with AEI/AE3 clone, Agilent) with chromogen streptavidin horseradish peroxidase and counterstain aminoethylcarbazine; bar $=20 \mu \mathrm{m}$

useful for distinguishing epithelial populations of cells. ${ }^{2,3}$ Vimentin is an intermediate filament that is present in most mesenchymal cells and is sensitive for most sarcomas and melanomas. ${ }^{2,3}$ Coexpression of vimentin and cytokeratin is possible with some thyroid, renal, and endometrial carcinomas, as well as mesothelioma. ${ }^{1}$ Overall, these stains are very sensitive for epithelial versus mesenchymal proliferations but are highly nonspecific. ${ }^{3}$ Due to the cytoplasmic uptake of pancytokeratin, this neoplasm was likely epithelial in origin.

Based on the history of an incompletely excised digital SCC, evidence of metastatic disease seen on thoracic radiography and contrast-enhanced CT, and cytologic assessment of both the fluid and lung nodules being consistent with SCC, the leading differential diagnosis was metastatic SCC in the thoracic cavity originating from an incompletely excised digital SCC of the pelvic limb. The time to metastasis was approximately 730 days from the time of initial diagnosis. The dog was euthanized at the referring veterinarian, and necropsy was not pursued.

\section{Interpretation and Case Summary}

Metastatic digital SCC diagnosed in the pleural effusion 730 days after incomplete excision of SCC of the fourth digit of the right pelvic limb in a dog.

\section{Comments}

Digital SCC is the most common malignant neoplasm affecting the digits of dogs, with the second most prevalent being digital malignant melanoma. In a retrospective study ${ }^{4}$ of 64 dogs with digital 
malignant neoplasms, SCC accounted for $51.6 \%$ of dogs and malignant melanoma accounted for $15.6 \%$, respectively. The median age of onset was 10 to 11 years, with large breed dogs having dark skin and coat color as a predisposing factor. Breeds predisposed to SCC include Standard Poodles, black Labrador Retrievers, Giant Schnauzers, Gordon Setters, and Rottweilers. ${ }^{5}$ There is a 2-fold higher incidence of thoracic limb digital SCC (66.9\%) over pelvic limb digital SCC (33.1\%). ${ }^{6}$

There are little published data on the incidence of metastasis of digital SCC in dogs. Overall, they have a propensity to be multicentric and tend to metastasize to lymph nodes and the lungs. A pool of several studies has reported that anywhere from $5 \%$ to $25 \%$ of digital SCC metastasize and the incidence varies on the location, time of diagnosis, treatment pursued, and whether there was bone involvement. ${ }^{6-10}$ In 1 study ${ }^{6}$ of 109 dogs diagnosed with digital SCC, the median time to metastasis was 309 days.

The possibility of a primary pulmonary SCC or a primary pulmonary adenosquamous carcinoma with subsequent carcinomatosis was considered. In this case report, the time to metastasis was 730 days, which is more than 2-fold the reported time to metastasis for digital SCC. In canine species, primary lung tumors are relatively rare and account for approximately $1.2 \%$ of all tumors. ${ }^{11}$ Pulmonary adenosquamous carcinomas account for $13 \%$ of all primary lung tumors, and given their rare occurrence are wholly understudied. ${ }^{12}$

In human literature, primary pulmonary SCC is the second most common type of primary lung tumor, pulmonary adenocarcinoma being most common, and accounts for $22.9 \%$ of all cases of non-small cell pulmonary neoplasia. ${ }^{13}$ In humans, as much as 91\% of pulmonary SCC has been attributable to cigarette smoking. Overall, the incidence of primary pulmonary lung tumors in humans is much higher than that of animals. ${ }^{14}$ Additionally, in canines, the incidence of metastatic tumors in the lung is much higher than that of primary lung tumors. ${ }^{14}$ Given the entire clinical picture, pulmonary metastatic SCC originating from the digit is most likely; however, other differential diagnoses must be given consideration in this case without subsequent necropsy and histopathology.

\section{References}

1. Dabbs D. Diagnostic Immunobistochemistry. 2nd ed. Churchill Livingstone Elsevier; 2006.

2. Bahrami A, Truong LD, Ro JY. Undifferentiated tumor: true identity by immunohistochemistry. Arch Patbol Lab Med. 2008;132:326-348.

3. Painter JT, Clayton NP, Herbert RA. Useful immunohistochemical markers of tumor differentiation. Toxicol Patbol. 2010;38(1):131-141. doi:10.1177/0192623309356449.

4. Henry CJ, Brewer WG Jr, Whitley EM, et al. Canine digital tumors: a Veterinary Cooperative Oncology Group retrospective study of 64 dogs. J Vet Intern Med. 2005;19:720-724.

5. Wobeser BK, Kidney BA, Powers BE, et al. Diagnoses and clinical outcomes associated with surgically amputated canine digits submitted to multiple veterinary diagnostic laboratories. Vet Patbol. 2007;44(3):355-361. doi:10.1354/ vp.44-3-355.

6. Belluco S, Brisebard E, Watrelot D, Pillet E, Marchal T, Ponce F. Digital squamous cell carcinoma in dogs: epidemiological, histological, and imunohistochemical study. Vet Patbol. 2013;50(6):1078-1082. doi:10.1177/0300985813490757.

7. Liu SK, Hohn RB. Squamous cell carcinoma of the digit of the dog. J Am Vet Med Assoc. 1968;153:411-424.

8. Marino DJ, Matthiesen DT, Stefanacci JD. Evaluation of dogs with digit masses: 117 cases (1981-1991). J Am Vet Med Assoc. 1995;207:726-728.

9. O'Brien MG, Berg J, Engler SJ. Treatment by digital amputation of subungual squamous cell carcinoma in dogs: 21 cases (1987-1988). J Am Vet Med Assoc. 1992;201:759-761.

10. Paradis M, Scott DW, Breton L. Squamous cell carcinoma of the nail bed in three related giant schnauzers. Vet Rec. 1989;125:322-324.

11. Brodey RS, Craig PH. Primary pulmonary neoplasmas in the dog; a review of 29 cases. J Am Vet Med Assoc. $1965 ; 147: 1628-1643$.

12. Hahn FF, Muggenburg BA, Griffith WC. Primary lung neoplasia in a Beagle colony. Vet Patbol. 1996;33:633-638.

13. Derman BA, Mileham KF, Bonomi PD, Batus M, Fidler MJ Treatment of advanced squamous cell carcinoma of the lung: a review. Transl Lung Cancer Res. 2015;4(5):524-532. doi:10.3978/j.issn.2218-6751.2015.06.07.

14. Wilson DW, Dungworth DL. Tumors of the respiratory tract. In: Meuten DJ, ed. Tumors in Domestic Animals. 4th ed. Iowa State Press; 2002:365-392. 